\title{
Distomatose et bilharziose des ruminants domestiques. Leur prophylaxie par la lutte anti-mollusques
}

\author{
por S. GRÉTILLAT
}

La distomatose des ruminants domestiques est une affection parasitaire presque cosmopolite causée par la présence dans les canaux biliaires de ces animaux d'un trématode du genre Fasciola Linné, 1758.

Alors que dans les pays tempérés, c'est en général Fasciola hepotica Linné, 1758, (grande douve), qui est en cause, c'est Fasciola gigantica Cobbold, 1855 (douve géante), que l'on rencontre en Afriqué tropicale, équatoriale et du sud, ainsi qu'aux Indes, en Indochine, à Sumatra, aux Philippines, aux lles Hawaï et dans le sud des Etats-Unis.

Ces deux parasites ont, comme tous les trématodes digénétiques, un cycle biologique passant par un mollusque hôte intermédiaire qui est en général un gastéropode d'eau douce du genre Lymnaea Lamarck 1779.

Nous rappellerons très brièvement, ici, les principales étapes de ce cycle qui a été étudié en détail pour la première fois en ce qui concerne F. hepatica, par THOMAS en 1881, puis la même année par LEUCKART.

Quant à F. gigantica, c'est ALICATA, (1938) aux lles Hawaï, puis DINNIK et DINNIK, (1958) au Kenya, qui on étudié sa biologie avec l'évolution de ses formes larvaires chez son mollusque vecteur.

Dans le genre Fasciolo, les ceufs pondus dans les canaux biliaires de l'animal parasité sont évacués avec les excréments ef doivent, pour pouvoir se développer, tomber dans l'eau douce. Dans ce milieu, le massif embryonnaire de l'œuf se transforme au bout d'un temps plus ou moins long, dépendant surtout de la température, en une larve ciliée appelée miracidium qui

Rev. Elev. Méd. vét. Pays trop. 1961, 14, no 3.

Reçu pour publication: Juin 1961. s'échappe de la coque de l'œuf en faisant sauter son opercule terminal.

Le miracidium nage jusqu'à ce qu'il rencontre l'hôte intermédiaire convenable qui est un mollusque.

A ce sujet il y a lieu de souligner l'extrême spécificité dans le choix du vecteur par la forme larvaire du trématode. C'est ainsi que toutes les espèces de Lymnaea ne sont pas réceptrices ( ${ }^{1}$ ).

Après avoir pénétré par l'orifice respiratoire du mollusque, le miracidium perd ses cils et se transforme en un sac à parois lisses contenant un massif cellulaire en voie de différenciation. C'est le sporocyste qui, au bout de deux à trois jours, va libérer par éclatement des formes larvaires, appelées rédies, présentant une ventouse antérieure avec un sac intestinal.

Par multiplication cellulaire interne, ces rédies vont produire d'autres jeunes rédies qui envahissent peu à peu tout l'hépato-pancréas du mollusque.

Après un certain temps d'évolution apparaissent, dans certaines de ces rédies, des formes larvaires constituées par une tête massive prolongćc par un appendice caudal simple et qui sont les cercaires.

Après avoir été libérées des rédies et avoir émigré dans les tissus périphériques de l'hôte intermédiaire, ces cercaires sortent du corps du mollusque quand ce dernier vient respirer à la surface de l'eau au cours d'une journée très ensoleillée. Elles nagent dans l'eau pendant un certain temps et vont s'enkyster sur des feuilles ou des tiges de végétaux aquatiques ou simple-

(1) Cas de Lymnaea hovorum (Tristram) à Madagascar, os l'invasion de l'île par la grande douve ne s'est pas produite malgré l'introduction répétée d'ovidés importés de France et à l'autopsie desquels il fut trouvé de très nombreuses F. hepstica. (Poisson, 1929). 
ment immergés accidentellement par une inondation des rives du cours d'eau.

Au cours de ce processus d'enkystement la cercaire perd sa queue, el ses cellules périphériques, ou batonnets cystogènes, se rassemblent pour lui constituer une coque protectrice lui permettant de résister aux conditions parfois difficiles du milieu extérieur (humidité, sécheresse, etc..) sans être détruite.

C'est en avalant ces formes de résistance appelées métacercaires, au cours du broutage des herbes découvertes par l'eau lors de la décrue, que les ruminants s'infectent. La coque de la métacercaire est digérée par les sucs digestifs, et le jeune organisme, qui possède déjà à l'état plus ou moins embryonnaire tous les organes de l'adulte, va se localiser dans les canaux biliaires de l'hôte définitif.

Nous n'insisterons pas sur les désordres et accidents morbides que peuvent déclencher la présence, en nombre parfois très important, de tels parasites dans le foie des animaux parasités amaigrissemnt, cachexie, œdème sous-glossien, anémie, lésions hépatiques telles que: angiocholite, sclérose hépatique, hémorragies, calculs, etc... et qui sont du domaine de la pathologie classique.

En ce qui concerne la bilharziose des ruminants domestiques, cette affection parasitaire est causée par un trématode de la famille des Schistosomidae Looss, 1899, qui est en général Schistosoma bovis (Sonsino, 1876), mais qui peut être Schistosoma japonicum Katsurada, 1904, en Extrême-Orient, ou bien Schistosoma spindale Montgomery, 1906, aux Indes et à Sumatra, ou Schistosoma indicum Montgomery, 1906, aux Indes ef en Rhodésie, ou Schistosoma curassoni Brumpt, 1931, au Mali, ou encore Schistosoma margrebowiei (Le Roux, 1933), en Rhodésie.

La valeur de ces trois dernières espèces ayan été pendant longtemps et encore très discutée, nous considérerons sculement le cas de la schistosomiase à Sch. bovis sensu stricto, dont la localication chez l'hôte définitif est le système veineux mésentérique.

Au point de vue de son cycle biologique, BRUMPT en 1929 et en 1930, l'a étudié en Corse où son hôte intermédiaire est Bulinus contortus.

Les femelles pondent des œufs à éperon terminal dans les fins capillaires de la paroi intestinale. Au bout de quelques jours passés dans l'épaisseur de la muqueuse de l'intestin, ces œufs à l'intérieur desquels se sont déjà développés des miracidia, tombent dans la lumière inteslinale d'où ils sont évacués vers l'extérieur avec les excréments. S'ils tombent dans l'ecu, la coque de l'œuf éclate dans les quelques minutes qui suivent, comme nous avons pu le constater expérimentalement au laboratoire, en travaillant sur des muqueuses d'ovidés parasités provenant de Mauritanie et abattus aux abattoirs de Dakar.

Les miracidia issus de ces éclosions sont très actifs, mais ne vivent qu'environ 6 heures. S'ils trouvent un mollusque réceptif, ils y pénètrent et se transforment en sporocystes donnant à leur tour des cercaires à queve bifide, ou furcocercaires, qui quittent l'hôte intermédiaire pour nager dans l'eau à la recherche de l'hôte définitif. Leur vie est très brève (quelques heures).

Au point de vue mode de pénétration de la furcocercaire chez le ruminant, BRUMPT (1930), parle de la voie transcutanée. Nous pensons, comme le supposent certains auteurs, qu'il s'agit plutôt de la voie digestive, surtout en ce qui concerne les bovidés. Chez ces derniers en effet, l'épaisseur et la dureté de la peau sont autant d'obstacles à la pénétration d'organismes aussi fragiles que les furcocercaires.

La schistosomiase bovine à Sch. bovis est en principe une parasitose des pays tropicaux et méditerranéens, la Corse (BRUMPT, 1930), la Sardaigne (BIOCCA et LEROUX, 1952), et la Sicile (GRASSI ef ROVELLI, 1888; BARBAGALLO, 1896), exceptées.

C'est ainsi qu'elle existe en Egypte, en Tunisie, au Soudan (Bamako: Rapport annuel, 1956), au Sénégal, en Guinée, en Haute-Volta, au Niger, au Dahomey, au Congo (Léopoldville : VAN DEN BERGHE, 1934), et en Afrique du Sud (PORTER, 1938 ; LE ROUX, 1929), si Sch. matthei Veglia et Leroux, 1929, n'est autre qu'un Sch. bovis.

A l'occasion d'une mission en Mauritanie, en février et mars 1960, dans la région de Kaédi, nous avons signalé l'existence ef l'importance de cette helminthiase sur les bovins ef les ovins.

Jusqu'à cette date la schistosomiase bovine ef ovine ne semble avoir été signalée que deux fois en Mauritanie :

1) Dans la région de Hodh (zovaza) (Rapport annuel du service de l'élevage de Mauri- 
tanie, 1952) sur des ovins en provenance de Nioro (Soudan-Mali).

2) Par une constatation d'abattoir faite par l'infirmier vétérınaire MOHAMED KALED sur des moutons de Mudjeria (région au Nord de Kaédi).

Nous insistons sur l'importance de cette cffection parasitaire en Mauritanie, car les constatations qu'a pu faire MARILL (1960 a), au cours d'une mission d'étude de 5 mois sur les schistosomiases humaines en République Islamique de Mauritanie, confirment celles que nous avons pu faire à Kaédi, où, sur 35 moutons, 5 chèvres et 3 bovins examinés, nous avons rencontré 18 cas de bilharziose animale, dont seulement 4 étaient bénins. MARILL signale en Mauriłanie 32,6 p. 100 et 67,3 p. 100 d'ovins et de bovins trouvés parasités aux abattoirs, avec très souvent des lésions généralisées aux viscères.

Comme le font remarquer la plupart des auteurs ayant étudié la question, et en particulier LE ROUX $(1929, b)$, ce n'est pas le schistosome lui-même qui est pathogène pour le ruminant, mais les complications et les lésions viscérales qu'il provoque.

Contrairement à ce que l'on observe dans la bilharziose vésicale humaine à Sch. haematobium (Bilharz, 1858), Sch. bovis ne provoque que très rarement de l'hématurie et encore moins souvent des hémorragies intestinales comme c'est le cas pour Schistosoma mansoni Sambon, 1907, chez l'homme.

Comme nous avons pu le constater à Kcédi en 1960, la schistosomiase animale se traduit plutôt par des symptômes généraux : tels qu'amai- grissement, cachexie, anémie, déréglement intestinal aboutissant à plus ou moins longue échéance à la mort de l'animal dans un état de misère physiologique extrême.

Les lésions observées à l'autopsie sont celles des grandes anémies, over ascite, couur flasque, léger hydropéricarde et oedème gélatineux péricardique. Le foie a un aspect «cuit», avec une surface bosselée. A la palpation il est dur, grumeleux, cirrhotique. Les poumons sont décolorés avec une pigmentation discrète donnant à l'organe un aspect plus ou moins grisâtre. Dans les très fortes infestations la muqueuse intestinale présente un léger piqueté hémorragique avec parfois légère congestion. Nous n'avons jamais remarqué d'hémorragie intestinale. Les seules lésions vésicales étaient représentées chez quelques animaux par de petits nodules hémorragiques, sans toutefois qu'il y ait présence de sang dans l'urine.

Nous nous sommes permis d'insister sur ce long préambule afin de faire ressortir toute l'importance que peuvent avoir chez les animaux domestiques, et en particulier, au Sénégal (MARILL, 1960, b ; GRETILLAT, 1960, d) et en Mauritanie, (MARILL, 1960, a : GRÉTILLAT, 1960 , d). la distomatose bovine ef la schisfosomiase des ruminants. Si ces deux affections n'ont pas le caractère spectaculaire de certaines maladies microbiennes ou à virus, elles portent un lourd préjudice à la vie économique de ces deux pays, en diminuant la valeur de leur cheptel dans une période où tous les moyens sont mis en cuvre pour développer le niveau de vie de leurs habitants.

\section{MOYENS DE LUTTE CONTRE LA DISTOMATOSE ET LA SCHISTOSOMIASE DES RUMINANTS DOMESTIQUES}

Ils sont de deux ordres ;

I) Moyens thérapeutiques : traitement des animaux malades par les procédés et produits offerts par la pharmacopée actuelle.

II) Moyens prophylactiques : A) Eviter l'infestation des animaux par les métacercaires (distomatose) ou par les furcocercaires (schistosomiase).

B) Couper le cycle évolutif du parasite, soit en empêchant ou en limitant la prolifération du mol- lusque vecteur hôte intermédiaire, soit en le détruisant par des moyens biologiques (prédateurs) ou chimiques (molluscocides).

\section{I) MOYENS THÉRAPEUTIQUES}

Malgré toute la valeur que peuvent présenter les produits antidistomiens ou antibilharziens mis au point ces toutes dernières années, des raisons d'ordre économique, et surtout pratique, 
limitent leur emploi à certains cas bien particuliers (troupeaux restreints et bien surveillés).

A l'échelle de l'Afrique et dans les conditions de l'élevage extensif, les distances et les difficultés rencontrées pour la contention des animaux rendent économiquement et pratiquement impensable un tel procédé de lutte.

\section{II) MOYENS PROPHYLACTIQUES}

Ce sont, à notre avis, les seuls pouvant donner des résultats appréciables avec des mises de fonds acceptables et un personnel réduit. II en est cependant qui sont difficilement applicables en Afrique.

\section{A) Eviter l'infestation des ruminants par les métacercaires ou par les furcocercaires.}

Le procédé suppose un contrôle des pâturages ou des abreuvoirs.

Il est inapplicable dans des pays où très souvent les pâturages infestés représentent la seule réserve d'herbe de fin de saison sèche, et où les points d'eau naturels sont rares et très éloignés les uns des autres. Pour ce dernier cas cependant, la création de puits, qui ne sont jamais des gîtes à mollusques, ef l'abandon des marigots et des mares infestés de schistosomiase, pourraient apporter une solution au problème. Mais cela suppose toute une éducation de la part des éleveurs qui répugnent, et on les comprend, à puiser à plusieurs mètres de profondeur, de l'eau que les animaux peuvent se procurer en surface.

\section{B) Couper ou perturber le cycle évolutif du trématode en agissant sur le mollusque hôte intermédiaire.}

a) Limifer ou empêcher la prolifération du vecteur.

Les conditions écologiques des mollusques d'eau douce étant parfois très strictes, il est possible, par des travaux d'assainissement, (drainage, assèchement de marais), de faire disparaître ou de limiter la prolifération de la faune malacologique de certains gîtes reconnus dangereux.

A ce sujet, il convient de rappeler combien il serait utile parfois d'associer à l'hydraulicien qui planifie l'aménagement des régions à mettre en valeur par l'irrigation, un écologiste et un épidémiologiste.

L'envahissement des canaux et des nouveaux plans d'eau par des mollusques vecteurs de maladies à trématodes pourrait ainsi être évité.

Il suffit, en effet, de prévoir certains aménagements peu coûteux dans les systèmes d'adduction d'eau, pour éviter de transformer une région saine en un secteur infesté de fasciolose ou de schistosomiase.

Au moment où dans tout l'ouest africain on cherche à mettre en valeur certaines régions par l'inlroduction de cultures irriguées (riz, coton), on devrait tenir compte des dangers que représentent, pour les santés humaine et animale, de tels travaux quand ils sont faits sans précautions spéciales. A ce sujet, on peut citer ce qui s'est récemment produit dans le sud tunisien pour la schistosomiase humaine, (COUMBARAS, 1960), où, à la suite de l'aménagement d'un réseau d'irrigation avec des conduites d'eau en ciment conçues d'une manière non adéquate, toute une région, jusqu'alors pratiquement indemne de bilharziose, a été envahie par le Bulin vecteur.

\section{b) Destruction du mollusque vecteur.}

Comme dernier moyen prophylactique d̀ mettre en œuvre dans la lutte contre les affections à trématodes, il reste celui qui est, à notre avis, le plus efficace dans les conditions représentées dans la plupart des régions en Afrique : la destruction du mollusque vecteur.

II va sans dire qu'on ne peut prévoir un programme d'assainissement massif de tous les gîtes à mollusques d'une région, et que toute action sur le terrain doit être précédée d'une ou plusieurs enquêtes malaco-épidémiologiques en vue de déterminer l'espèce vectrice et de recueillir le maximum de renseignements sur son écologie.

Si, en effet, il est toujours possible de supprimer ou, tout au moins, de diminuer la faune malacologique d'un gîte, l'opération sera d'autant moins onéreuse qu'elle sera plus efficace, faite à une époque où l'hôte intermédiaire sera plus facilement accessible, plus vulnérable et plus rare, dans un volume d'eau réduit, avant cependant qu'apparaissent certaines formes de résistance, qui, en assurant la survie de l'espèce 
d'une saison à l'autre, la rend invulnérable aux différents moyens utilisés pour la détruire.

1) Destruction des mollusques por des procédés d'ordre biologique.

On a beaucoup écrit sur le déséquilibre biologique du milieu pour lutter contre les mollusques vecteurs.

L'introduction d'animaux prédateurs tels que les canards et les oies, qui sont de grands mangeurs de mollusques, a été préconisé. Aucune expérience valable ne semble avoir été faite sur le terrain et les auteurs se sont contentés d'extrapoler les résultats obtenus dans une volière, à ceux que l'on pourrait obtenir sur le terrain. II semble qu'il ne faille pas attendre beaucoup de chose de cette méthode, car un oiseau a un comportement tout autre selon qu'il est en captivité et nourri artificiellement, ou en liberté en face d'une nourriture variée qu'il peut choisir.

Certains poissons sont malacophages, comme I'a montré LAGRANGE en 1953, mais les conditions d'acclimatement de ces prédateurs limitent les chances de succès de cette méthode de lutte biologique.

En ce qui concerne les crustacés, les écrevisses du genre Astacus et Cambarus, ainsi que le crabe Potamon edule ont été reconnus comme de grands prédateurs de mollusques d'eau douce (DESCHIENS et LAMY, 1953 ; DESCHIENS, 1954 ; DESCHIENS ef LAMY, 1954 ; DESCHIENS, DESCHANGE et VERMEIL, 1955).

CHERNIN, MICHELSON ef AUGUSTINE, en 1956, signalent que la sangsue Helodello fusca attaque et détruit les formes adultes de Planorbes.

Dans un autre ordre d'idée, et en faisant intervenir la fréquence du parasitisme à Echinostomidae (Trematoda) de la plupart des oiseaux sauvages aquatiques, BAYER en 1954, parle de la possibilité de bouleverser le parasitisme larvaire des mollusques vecteurs d'un gîte, en favorisant et en protégeant la multiplication de ces volatiles. D'après cet auteur, en infestant massivement les mollusques par des formes larvaires d'échinostomes, ces oiseaux « mobiliseraient», en quelque sorte, toute la faune malacologique vectrice, qui de ce fait, ne serait plus « réceptrice » aux miracidia des trématodes de l'homme et des animaux domestiques.

Les constatations que l'on peut faire sur le terrain à la dissection des mollusques trouvés dans un gîte, ainsi que les expériences d'infestation expérimentale réalisées au laboratoire, montrent que, biologiquement, un tel bouleversement parasitaire des vecteurs est difficilement réalisable. Les conditions qui président à l'« acceptation » du miracidium par le mollusque créent, en effet, un équilibre dans les différents pourcentages de la nature de l'infestation des mollusques.

D'autres auteurs ont pensé que la modification de la faune microbienne des gîtes pourrait apporter un déséquilibre biologique amenant la disparition de sa faune malacologique. C'est ainsi que DIAS, en 1953 et 1954, DIAS et DAWOOD en 1954, ont essayé au laboratoire de détruire Australorbis glabratus (Say) en provoquant une prolifération intense du Bacillus pinotti ou Bacille B.E.T., qui est mortel pour ce mollusque.

Au point de vue équilibre biologique des espèces de mollusques se trouvant dans un gîte, ALVES en 1956, à la suite d'observations faites sur le terrain, a remarqué une disparition temporaire des Physopsis consécutive à la prolifération intense de Bulinus, mais ce ne fut que temporaire, et il semble que l'on ne puisse en tirer des conclusions d'ordre pratique.

A Puerto-Rico OLIVER-GONZALEZ, BAUMAN ef BENENSON ont signalé en 1956, l'extrême voracité de l'operculé Marisa cornuarietis qui d'après ces auteurs est capable de diminuer considérablement la densité des Planorbes d'un gîte en raréfiant la nourriture.

Malgré tout l'intérêt et la valeur que présentent tous ces travaux, il est difficile d'extrapoler leurs résultats, qui sont pour la plupart expérimentaux, à une application sur le terrain, où l'on se trouve en présence de facteurs entravant le développement normal du prédateur, ou annulant partiellement son activité prédatrice.

il est néanmoins possible, sinon probable, qu'à la suite des nombreuses recherches déjà en cours sur l'écologie des mollusques vecteurs, on arrive à mettre au point des moyens de lutte biologique

Ils seront cependant toujours plu's ou moins limités à certains biotopes dont les conditions bio-physico-chimiques seront parfaitement connues, et ne pourront être généralisées à l'ensemble des gîtes à mollusques d'une région à assainir. 
2) Destruction des mollusques par des produits chimiques.

C'est de nos jours le procédéle plus employé et le plus efficace.

Au cours de cette dernière décade, il a fait l'objet de très nombreuses recherches dans tous les pays où les affections parasitaires à trématodes revêtent un caractère important ou parfois alarmant.

La question des produits molluscocides dans la prophylaxie des maladies a trématodes est tellement importante que l'Organisation mondiale de la Santé à Genève a créé, depuis quelques années déjà, un Comité d'experts des bilharzioses dont un groupe ne s'occupe que des produits antimollusques (recherche de nouveaux produits, expérimentation au laboratoire et sur le terrain, études sur l'apparition de phénomènes de résistance de la part des mollusques, etc...).

Cet organisme essaye depuis quelques temps de normaliser, autant que faire se peut, les techniques de contrôle en même temps que de coordonner et de diffuser les résultats obtenus par les différents laboratoires travaillant sur les produits antimollusques.

D'après l'Organisation mondiale de la Santé, le molluscocide idéal devrait réunir les conditions suivantes:

10 Actif contre les mollusques vecteurs et contre leurs pontes à des doses inférieures ou voisines de 10 parties par million (au laboratoire, la dose létale 100 devant être obtenue en $24 \mathrm{~h}$ de contact, suivies d'un temps de lavage ou de réanimation de $48 \mathrm{~h}$ ). Le mollusque de référence devant être autant que possible Biomphalaria pfeifferi ou Australorbis glabratus. Ces deux espèces considérées comme étant très résistantes à l'action des molluscocides.

$2^{\circ}$ Très diffusible pour ne pas nécessiter une agitation du milieu, et ne pas obliger à un faucardage préalable des plantes aquatiques encombrant le gîte.

3o Présentant une activité conservée ou très peu influencée par des $\mathrm{pH}$ allant de 5 à parfois 8,5 , et des températures variant entre $+15^{\circ} \mathrm{C}$ ef $+35^{\circ} \mathrm{C}$.

40 Résistant, ou très lentement détruit par la lumière solaire (Rayons ultra-violets). (Rémanence d'action d'au moins 24 heures).
$5^{0}$ Activité conservée dans des milieux très boueux et fortement chargés en matières organiques.

$6^{0}$ Très peu absorbé par les plantes aquatiques.

70 Non toxique pour l'homme et les animaux domestiques aux doses employées pour la destruction des mollusques.

$8^{\circ}$ D'utilisation simple et facile sans présenter de danger pour les utilisateurs.

9o Non toxique, ou très faiblement toxique pour la faune aquatique. (Poissons, batraciens, crustacés, insectes, etc...).

Condition essentielle pour ne pas bouleverser l'équilibre biologique du milieu et ne pas priver de protéines les populations riveraines vivant des produits de la pêche.

$10^{\circ}$ D'un prix de revient abordable de manière à pouvoir être utilisé sur une grande échelle.

110 Stable ef de conservation facile dans les conditions de stockage rencontrées sous les climats tropicaux.

Un tel enisemble de conditions est difficile d̀ réaliser, et à l'heure actuelle on peut dire qu'aucun des molluscocides employés ou en cours d'expérimentation, ne répond à de telles exigences.

Le molluscocide idéal reste donc encore à trouver.

Au cours des lignes qui vont suivre nous passerons en revue les principaux antimollusques employés ou à l'essai dans le monde, et faisant l'objet d'un contrôle direct ou indirect de la part du Comité d'Experts de la Bilharziose, Section Molluscocides, de l'Organisation Mondiale de la Santé à Geriève.

Ce seront les sels solubles de cuivre, les pentachlorophénates, les dinitrophénols, les sels de baryum, le Bayer 73 ou 2-dichloro-4-nitro-anilide-5-chlorosalicylique, l'acroléine, ou Aqualin Shell, et quelques autres de moindre importance ou dont les résultats ne sont encore qu'imparfaitement connus et qui sont : le «Méta » ou métaldéhyde éthylique, et le ICl 24223.

Après quoi nous aborderons l'exposé des travaux réalisés au Laboratoire national de reherches vétérinaires de Dakar avec un nouveau produit antimollusque, le diméthyldithiocarbamate de zinc ou zirame. 


\section{SELS SOLUBLES DE CUIVRE}

Quoique le cuivre métal présentc une haute toxicité pour les mollusques d'eau douce, (LAGRANGE, 1951), ce sont les sels solubles de cuivre qui sont couramment utilisés comme molluscocides, les sels insolubles tels que le carbonate, ou le sulfate basique de cuivre n'agissant que s'ils sont absorbés par les mollusques.

Parmi les sels solubles c'est le sulfate de cuivre technique qui pendant de très nombreuses années a été le seul molluscocide valable présentant un intérêt pratique.

L'acétate neutre de cuivre, comme nous avons pu le constater au laboratoire, aurait peut-être une activité légèrement supérieure à celle du sulfate, mais il présente les mêmes inconvénients et il est plus chcr. Son cmploi ne présente donc aucun intérêt pratique.

II n'est pas dans notre intention de rappeler ici les très nombreux travaux réalisés sur les propriétés antimollusques du sulfate de cuivre. Nous voudrions cependant fairc remarquer que si ce produit est actuellement quelque peu délaissé au profit d'autres plus récents, il n'en est pas moins encore utilisé au Soudan (Khartoum) (MALEK, 1960, a), en Egypte (EL GINDY, 1958) et au Brésil (AGUIRRE, SYLVA et TAVARES, 1955), où il rend de très grands services dans la lutte contre les bilharzioses.

En effet, dans certaines conditions d'emploi, il reste le seul composé chimique dont le choix s'impose pour une campagne anti-mollusques.

Voici brièvement exposés, les avantages ef les inconvénients qu'il présente et qui motivent ou qui rendent impossible son emploi dans les conditions de la pratique.

Avantages : Il est pratiquement sans toxicité pour l'homme et les animaux domestiques. II est bon marché. II peut être facilement répandu dans les gîtes à mollusques par un personnel non spécialisé (Cristaux mis à fondre dans des sacs de jute placés dans le courant (rivières) ou traînés le long des berges s'il s'agit d'une collection d'eau sans courant appréciablc.

Aux doses actives contre les mollusques, il est très peu toxique pour la faune et la flore aquatiques.

Inconvénients : II flocule et précipite dans les eaux dont le $\mathrm{pH}$ est supérieur à 7 .
Il est très fortement ef très rapidement absorbé par les matières organiques avec lesquelles it forme des complexes chimiques insolubles et inactifs contre les mollusques.

Il est fixé par les végétaux aquatiques et diffuse très mal dans les eaux encombrées par ces plantes, de sorte qu'il est la plupart du temps nécessaire de faire procéder à leur faucardage, (opération lente et onéreuse), avant le traitement du gîte.

In vitro, et en eau claire, il tue la plupart des mollusques d'eau douce à des concentrations de 0,5 à 1 partie par million, alors que sur le terrain il est nécessaire de répandre 10, 20, 30 et parfois 100 parties par million pour obtenir des effets identiques.

Devant les quantités parfois énormes de sulfate de cuivre perdues par fixation sur l'argile ef la vase des fonds, MALEK $(1960$, b) pense qu'il serait peut-être intéressant de savoir dans quelle mesure on pourrait récupérer tout ce cuivre inactivé en le libérant au moyen de chélateurs (saponines par exemple). Mais cet auteur pense que les quantités de saponines nécessaires à une telle opération seraient un obstacle économique pour n'aboutir peut-être qu'à des résultats décevants.

Pour éviter la floculation du sulfate de cuivre dans les eaux alcalines, inconvénienf signalé en particulier par WOLFS et DEVIGNAT (1949) à Bukavu (Congo Léopoldville), certains auteurs ont essayé de l'associer à divers acides organiques.

En Egypte, EL GINDY (1953) obtient de bons résultats dans les eaux du Nil en ajoutant aux 30 p.p.m. de sulfate de cuivre répandus, 5 p.p.m. d'acide tartrique. D'après le même auteur l'acide citrique serait préférable à l'acide tartrique.

Por confre, MAGALAHES, MORAES, ALMEIDA et CAIADO (1953) prétendent que l'acide tartrique diminue l'activité molluscocide du sulfate de cuivre.

Personnellement nous avons fait au Laboratoire national de recherches vétérinaires de Dakar des tests de comparaison « in vitro » sur Biomphalaria pfeifferi gaudi Ranson, et Bulinus guernei Dautzemberg, avec sulfate de cuivre seul à 1 p.p.m. et sulfate de cuivre à 1 p.p.m. + acide tartrique à 0,2 p.p.m., sans observer une diminu- 
tion d'activité appréciable dans les aquariums contenant de l'acide tartrique.

\section{LES PENTACHLOROPHÉNATES}

Ce sont actuellement les molluscocides les plus employés dans le monde. On utilise soit le pentachlorophénate de sodium, soit le pentachlorophénate de cuivre.

\section{PENTACHLOROPHÉNATE DE SODIUM}

Avantages : produit bon marché et d'un maniement relativement facile surtout si on l'utilise sous forme de briquettes.

Soluble dans l'eau, il est très toxique pour les mollusques d'eau douce, et dans les conditions de la pratique il les détruit à des concentrations allant de 10 à 15 p.p.m. en rivière (DOBROWOLNY et BARBOSA, 1953), ct sculement 5 p.p.m. dans des gîtes où l'eau est calme (mares).

Même à faible concentration il détruit les œufs de Planorbes (OLIVER et HASKINS, 1960).

Les eaux traitées ne présentent aucun danger pour l'homme et les animaux domestiques (HERDT, LOOMIS et NOLAN, 1951).

Quand les gîtes ne sont pas trop encombrés par les plantes aquatiques il n'y a pas lieu de faire procéder à un faucardage préalable.

Inconvénients : Si ce produit est d'une manipulation facile, (briquettes solubles), il y a lieu cependant de prendre certaines précautions lors de son emploi sous forme de poudre à mettre en solution avant le traitement. Comme le signalent BARNES (1960) et BLAIR (1960) le contact du produit ou des solutions concentrées avec la peau ou les muqueuses; au niveau desquelles il est très rapidement absorbé, peut provoquer des accidents mortels.

Quand il est utilisé dans des points d'eau très chargés en matières organiques et très vaseux, les concentrations initiales du produit diminuent très rapidement car il est fixé par ces matières. 10 parties par million seraient réduites à 1 p.p.m. au bout de 8 heures selon DOBROVOLNY et HASKINS (1953).

Il en est de même si la flore aquatique est très dense, et un faucardage préalable s'avère nécessaire si on désire avoir une bonne dispersion du produit. (Observations faites par GILLET, BRU-
AUX et NANNAN, 1960, dans la vallée de la Ruzizi au Kivu (Congo-Léopoldville).

La lumière solaire (rayons ultra-violets) le détruit aussi rapidement ( 8 heures: DOBROVOLNY et HASKINS, 1953) et on a même pensé pour éviter cet inconvénient à traiter les gîtes durant les dernières heures de la journée.

D'autre part, quand le pentachlorophénate de sodium est répandu au cours d'une journée très chaude, les couches supérieures de l'eau étant plus chaudes que les inférieures, le molluscocide a tendance à se répandre suivant des strates correspondant aux différences de température du milieu, et l'on est obligé d'agiter l'eau pour assurer une dispersion uniforme du produit (BERRY, 1960).

Au point de vue faune aquatique, il tue les poissons, les batraciens et les crustacés aux doses utilisées contre les mollusques (GILLET, 1960). II est d'autre part légèrement herbicide et son emploi ne serait pas indiqué dans le traitement de rizières.

Malgré tous ces inconvénients, le pentachlorophénate de sodium (PCPNa), continue à être le molluscocide le plus actif et le plus employé dans la lutte contre les bilharzioses au Brésil (PAULINI, 1956), en Afrique du sud (SHIFF, 1960), au Venezuela (JOVE, 1956), en Egypte (NAGUIB, 1960), aU Japon (KOMYA, HOSAKA, YASUORAKA, 1960), aux Philippines (RITCHIE ef MC MULLEN, 1960) et d'autre part en Allemagne pour la prophylaxie de la distomatose des ruminants (ENIGK, 1956 ; ENIGK et DUWEL, 1960).

\section{PENTACHLOROPHÉNATE DE CUIVRE}

C'est un molluscocide d'une très grande efficacité. Il est insoluble dans l'eau et peut ainsi être ajouté soit à du sulfate de cuivre, soit à du pentachlorophénate de sodium. Son prix de revient est relativement faible et d'après les résultats des essais faits au Vénézuéla, ses effets seraient plus durables que ceux des deux produits précités. JOVE (1956) aurait eu de bons résultats en l'utilisant à 15 p.p.m. pendant 6 heures dans des gîtes à Planorbes.

\section{LES DINITROPHÉNOLS}

Parmi ces composés chimiques, ce sont surtout le Dinitro-o-cyclohéxylphénol (D. C. H. P.) 
et l'un de ses sels, la Dicyciohéxylamine, qui ont été reconnus comme ayant d'intéressantes propriétés molluscocides.

KUNTZ etSTIREWALT (1950) puis KUNTZ et WELLS, ont démontré qu'à des concentrations de 3 à 5 p.p.m., le DCHP était actif in vitro contre Biomphalaria sp. et Bulinus sp.

C'est un produit bon marché qui n'est pas toxique pour l'homme et les animaux domestiques aux doses utilisées contre les mollusques.

II n'est pas phytotoxique, mais il est par contre très ichtyocide.

Les recherches ont été menées en ExtrêmeOrient dans la lutte contre les Onchomelania vecteurs de Schistosoma japonicum (PESIGAN MASILUNGAN, 1950 ; WILLIAMS, NOON, OTORI, FRICK et RITCHIE, 1955).

Sur le terrain, dans des gîtes très encombrés par des plantes aquatiques, HALAWANI (1954) a trouvé à l'aide d'un procédé de dosage colorimétrique que l'eau, traitée avec 10 p.p.m. de DCHP, n'en renfermait plus que 3 p.p.m. au bout de 24 h., et 1 p.p.m. au bout de 6 jours. D'après cet auteur, ce produit serait légèrement ovicide pour les pontes de mollusques.

II semble que la grande toxicité de ces produits pour les poissons soit un des principaux obstacles à leur utilisation dans la pratique courante.

\section{LES SELS DE BARYUM}

C'est NOLAN, BOND et MANN, en 1953, qui semblent avoir signalé les premiers l'action molluscocide des sels de baryum; en l'occurrence. il s'agissait d'un sel de baryum du pentachlorophénol.

Puis des travaux sur les sels solubles de ce métal (chlorure, nitrate) et insolubles (carbonate) ont été réalisés par BIJAN et DESCHIENS (1956).

Des recherches plus récentes (COROLLER, 1960), faites en Egypte en utilisant pour les tests de laboratoire les eaux du Nil, d'une part, et l'eau ordinaire d'autre part, montrent que le chlorure de baryum tue Biomphaloria boissyi en 48 h. à raison de 20 p.p.m. ; en 36 h. à 50 p.p.m. ; en 24 h. à 80 p.p.m. ; et pratiquement en 12 h. à 100 p.p.m.

Ce produit qui est relativement bon marché, serait peu toxique pour les poissons et, aux doses ci-dessus indiquées, ne présente aucun danger pour l'homme et les animaux domestiques susceptibles d'utiliser les eaux traitées.

En ce qui concerne le carbonate de baryum qui est presque insoluble dans l'eau, il s'avère être toxique pour les Planorbes. Selon COROLLER, il se transformerait en chlorure de baryum toxique dans le tube digestif de ces mollusques, mais pratiquement cela suppose que les Biomphalaria avalent le produit, ce qui est à notre avis tout à fait aléatoire.

Aucune expérience ne semble avoir été faite sur le terrain avec ces produits. On n'a donc pour l'instant aucun renseignement quant à leur pouvoir dispersif, leur rémanence dans des gîtes très chargés en matières organiques et leur pouvoir ovicide sur les ceufs de mollusques.

Cependant les premiers résultats obtenus au laboratoire avec les sels solubles de baryum permettent d'espérer que, dans les années à venir, ces produits, et en particulier le chlorure de baryum rendront de grands services dans la lutte contre les affections à trématodes de l'homme et des animaux domestiques (DESCHIENS, AYAD ef le CORROLLER, 1960).

\section{LE BAYER 73 OU (2-DICHLORO-4-NITRO) ANILIDE-5-CHLOROSALICYLIQUE}

C'est en 1958, au Congrès international de médecine tropicale de Lisbonne que GONNERT et SCRAUFSTAETTER ont présenté pour la première foisce produit antimollusque fabriqué par la firme Bayer et commercialisé sous le nom de Bayer 73.

Depuis lors, les essais, faits avec ce produit tant au laboratoire que sur le terrain, ont démontré son haut pouvoir molluscocide même à faible concentration, 0,3 à 0,5 p.p.m.

Le gros travail de GONNERT (1960) a permis de faire le point en ce qui concerne les recherches effectuées depuis 1958 avec ce produit.

Si quelques petites différences existent entre les résultats obtenus au laboratoire par les différents chercheurs ayant testé le Bayer 73 , sur le terrain tous les expérimentateurs sont d'accord pour lui accorder les avantages suivants :

Actif à des doses très faibles, il n'exige le transport sur les lieux de traitement que de petites quantités de produit, ce qui est un gros avantage en Afrique. 
II est pratiquement sans danger pour l'homme ef les animaux domestiques.

Son activité n'est pas influencée par le $\mathrm{pH}$ du milieu (activité optimum par un $\mathrm{pH}$ de 7,8 ), qui peut être très boueux et très chargé en matières organiques.

Il diffuse bien dans les gîtes encombrés par des plantes aquatiques.

II n'a que peu d'effet sur la végétation aquatique et il est vraisemblablement très lentement absorbé par les matières organiques.

II détruit les pontes de mollusques (FREITAS, 1959).

En résumé, il présente à peu près tous les avantages du pentachlorophénate de sodium sans en présenter certains inconvénients, tout en agissant à des doses 10 à 20 fois plus faibles.

Malheureusement, et tous les auteurs le signalent, il est très toxique pour la faune aquatique à des concentrations voisines de celles actives contre les mollusques.

WEBBE, 1960, a constaté dans des étangs traités avec Bayer 73 une importante mortalité chez Tilapia esculenta (Graham), T. melanopleura (Dumerie), Protopterus aethipicus (Heck), Clarias nossambicus (Peters) et Astatorochromis alluaudi (Pellegr.) dans los 21 h. D'autre part, un nombre important de grenouilles qui avaient quitté les étangs quelques heures après le traitement furent trouvées mortes sur les rives.

GILLET, BRUAUX et NANNAN (1960) ont fait des constatations identiques dans la vallée de la Ruzizi, au Kivu, ef FOSTER, TEESDALE et POULTON (1960) signalent l'ichtyotoxicité du Bayer 73 au cours d'essais faits au Kenya.

En ce qui concerne la résistance du produit aux R. U. V. GILLET et BRUAUX (1960) ont montré qu'en 24 h une solution à 0,5 p.p.m. était réduite au $1 / 11$ e et qu'une solution à 0,25 p.p.m. était réduite dans le même temps au $1 / 6^{\mathrm{e}}$ pour une température de 24-260.

SHIFF, 1960, fait les mêmes remarques sur le terrain au sujet de l'influence de la lumière solaire sur le Bayer 73.

En conclusion, si ce n'est sa haute toxicité pour la faune aquatique, ce molluscocide est remarquable par sa grande activité à faible concentration ef par son omnivalence quant aux conditions physico-chimiques présentées par le milieu à traiter.

\section{ACROLÉINE (AQUALIN SPELL) \\ QU HERBICIDE SHELL F 98}

Ce produit commercialisé par la Shell Chcmical Corporation, est un herbicide puissant contre les plantes aquatiques des genres Potamogeton, Hydrodictyon, Cladophora, Spyrogyra, Zandichiella, Elodea, Callitriche, Ceratophyllum, Chara et Compsapogon, à des doses variant entre 1,5 et 7,5 p.p.m.

Avec des concentrations de 15 à 20 p.p.m. minimum, il provoque le dessèchement ef la mort des plantes flottantes des genres Pistia, Eichornia et Jussiaea, qui envahissent la surface de certains plans d'eau. II n'a par contre aucune action toxique sur des plantes telles que le blé, le millet, le coton, les tomates qui peuvent sans danger être irriguées avec l'eau traitée par ce produit, à des doses de 80 p.p.m. (Rapport Shell Devel. Co., 1958).

FERGUSON, RICHARDS et PALMER, 1960, à Porto-Rico, ont montré qu'au laboratoire, l'Aqualin tue Australorbis glabratus en $24 \mathrm{~h}$ à des concentrations de 2,5 à 5 p.p.m. et en 3 h. à des concentrations de 10 p.p.m. Sur le terrain 3 à 5 p.p.m. semblent être suffisantes pour tuer A. glabratus et les mollusques uperculés du genre Marisa.

Dans des rivières de Porto-Rico, son pouvoir de diffusion qui semble augmenter avec la température du milieu a permis de constater qu'il tuait les mollusques à plus d'un kilomètre et demi do distance de son point d'application.

VAN OVERBEEK, HUGUES ef BLONDEAU, 1959, estiment qu'il est deux fois plus actif que le pentachlorophénate de sodium et qu'il est 'plus toxique pour les œufs de mollusques que le sulfate de cuivre: 100 p.p.m. tueraient 100 p. 100 des œufs en 24 heures (FERGUSON, RICHARDS ef PALMER, 1960).

II ne serait que très lentement fixé par les matières organiques.

Malheureusement, c'est un produit qui est d'une manipulation extrêmement délicate et dangeureuse et qui ne peut être employé que sous la surveillance de spécialistes et avec un équipement adéquat. II est en effet extrêmement corrosif et irritant pour les muqueuses.

D'autre part, si les equx traitées ne présentent aucun danger pour l'homme ef les animaux domestiques, l'Aqualin est toxique pour la faune aquatique (FERGUSON, RICHARDS et PALMER 1960). 
Ces deux inconvénients sont deux grands handicaps pour l'utilisation d'un produit qui présente pourtant l'avantage d'être à la fois herbicide et molluscocide.

\section{AUTRES MOLLUSCOCIDES ACTUELLEMENT EN COURS D'ESSAI}

Parmi eux nous ne citerons que $\mathrm{ICl} 24223$ et le métaldéhyde ou « Méta ».

Le $\mathrm{ICl} 24223$ a été essayé au Mozambique en solution dans le toluène. Dans les eaux calmes il agirait à des doses voisines de 0,4 p.p.m.

Les résultats obtenus avec ce produit sont encore trop fragmentaires pour permettre de tirer des conclusions quant à ses avantages ef ses inconvénients.

Le métaldéhyde éthylique ou « Méta », ou alcool solidifié, qui est couramment utilisé dans la lutte contre les limaces des jardins, s'est révélé toxique pour Bulinus contortus et Planorbis glabratus comme l'ont montré DESCHIENS et MOLINARI en 1956.

Il est non soluble dans l'eau et doit être répandu sous forme de poudre à la surface du gîte. II agit par contact et par ingestion, mais contrairement à ce qui se passe pour les limaces, il n'a aucun pouvoir attractif pour les mollusques d'eau douce qu'il tuerait en 5 à 36 heures.

Récemment, ENICK et DUWELL en 1960, l'ont essayé pour lutter en Allemagne contre Lymnaea truncatula qui estl'hôte intermédiaire de la grande douve en Allemagne.

Non toxique pour les poissons, les gros crustacés, les batraciens et les plantes aquatiques, il ne présente aux doses employées aucun danger pour l'Homme et les animaux domestiques. Cependant à des doses per os de 0,15 à $0,5 \mathrm{gr}$ par jour, il est légèrement hypnotique pour l'homme.

Malheureusement c'est un produit relativement cher et son insolubilité alliée au fait qu'il ne peut agir sur les mollusques que par contact ou par ingestion, limite considérablement son emploi dans la pratique courante.

\section{ESSAIS FAITS AVEC LE DIMÉTHYLDITHIOCARBAMATE DE ZINC OU ZIRAME}

Le diméthyldithiocarbamate de zinc ou zirame est un produit utilisé ef commercialisé à l'heure actuelle comme fongicide dans la lutte contre les maladies cryptogamiques des végétaux.
C'est le plus stable des diméthyldithiocarba mates métalliques fongicides.

A la température ordinaire, il se présente sous la forme d'un solide sans odeur, sans goût et non corrosif.

Sa formule est la suivante: $\left(\left(\mathrm{CH}^{3}\right) 2 \mathrm{NCS}\right) 2$ $\mathrm{Zn}$, et son poids moléculaire est de 305,8 .

Avec un point de fusion de $246^{\circ} \mathrm{C}$., il a une densité voisine de 2 à $20-24^{\circ} \mathrm{C}$.

II est soluble dans le chloroforme mais insoluble dans l'éther et dans l'alcool.

II est faiblement soluble dans l'eau $(65 \mathrm{mg} /$ litre).

Irrilant pour les yeux et les muqueuses, il est inflammable, comme la plupart des dérivés soufrés, et il y a lieu de ne pas le placer à proximité d'une flamme.

Travaux déjà réalisés sur l'action oligodynamique du zinc ou de ses dérivés contre les mollusques d'eau douce.

Au même titre que l'ion cuivre, l'ion zinc est toxique pour les mollusques d'eau douce sur lesquels il a cependant une action oligodynamique moins marquée.

Comme nous avons pu le constater au laboratoire, en utilisant comparativement des solutions de sulfate de cuivre et de sulfate de zinc, alors qu'il suffit d'une concentration de 1 p.p.m. de $\mathrm{SO}^{4} \mathrm{Cu}$ pour tuer Biomphalaria pfeifferi gaudi en 24 heures, il est nécessaire d'utiliser 20 p.p.m. de $\mathrm{SO}^{4} \mathrm{Zn}$ pour obtenir les mêmes résultats.

C'est DESCHIENS et MOLINARI en 1957 (a), qui démontrèrent pour la première fois l'action oligodynamique de ce métal pour les mollusques d'eau douce en utilisant l'oxyde de zinc contre Planorbis glabratus et Bulinus contortus.

C'est bien le zinc qui est toxique puisque la même année, (1957, b), ces mêmes auteurs, puis DESCHIENS, MOLINARI et BERTRAND (1957) obtinrent des résultats positifs avec la grenaille de zinc.

Ces expériences avaient été dictées par les résultats obtenus en 1955 par NOLAN et BOND avec un dérivé du zinc, le diméthyldithiocarbamate de zinc.

$\mathrm{Ce}$ produit, que ces auteurs considérèrent d'emblée comme un «potential molluscicide», tuait Australorbis glabratus en 24 heures de contact pour une concentration de 1,5 p.p.m.

II faut attendre 1960 pour que des essais avec 
ce produit soient faits par PAULINI, CHAIA et FREITAS au Brésil.

Pour leurs expériences au laboratoire et sur le terrain, ces auteurs utilisèrent des comprimés et une poudre renfermant 50 p. 100 de zirame et 50 p. 100 de carbonate de calcium.

A la dose de 5 p.p.m. ce mélange se montra à 100 p. 100 actif contre Australorbis glabratus, avec une bonne rémanence de plusieurs semaines sur le terrain. Cependant d'après le compte rendu des résultats obtenus, il semble que ce mélange soit difficilement soluble dans l'eau.

Essais faits au Laboratoire national de recherches vétérinaires de Dakar avec le diméthyldithiocarbomate de zinc.

Au cours d'une série d'essais faits au laboratoire avec un certain nombre de produits molluscocides tels que le sulfate de cuivre, l'acétate neutre de cuivre, le sulfate basique de cuivre, le Bayer 73, nous avons été amené à examiner l'activité molluscocide du zirame.

Nous avons réalisé ces expériences à l'aide d'une poudre micronisée titrant 90 p. 100 de diméthyldithiocarbamate de zinc, dont 100 p. 100 des particules présentent un diamètre inférieur à $40 \mu$, parmi lesquelles 90 p. 100 ont un diamètre inférieur à $10 \mu$. Cette présentation, un peu particulière, est celle mise au point pour être utilisée dans la lutte contre les champignons parasites des végétaux, qui nécessite un emploi sous forme de bouillie, à répandre par pulvérisation.

\section{Matériel utilisé :}

Les tests in vitro ont été faits sur des mollusques d'élevage appartenant aux espèces suivantes :

Biomphalaria pfeifferi gaudi Ranson, Bulinus senegalensis Müller, Lymnaea caillaudi Bourguignat ef Bulinus guernei Dautzemberg, gastéropodes d'eau douce les plus fréquemment rencontrés dans les mares, marigots et rivières au Sénégal.

C'est l'homogénéité de ce matériel au point de vue résistance qui nous a fait préférer des mollusques d'élevage à des mollusques récoltés dans des gîtes naturels.

Parmi ces derniers, en effet, il existe toujours un certain pourcentage de spécimens infestés par des formes larvaires de trématodes, qui sont beaucoup plus sensibles aux produits anti-mollusques que les spécimens non infestés. Ces différences de résistance ne peuvent que fausser les résultats de l'expérimentalion.

\section{Protocole expérimental :}

Les tests d'activité in vitro sont faits sur 30 spécimens de chaque espèce (adultes ayant de 2 à 3 mois d'âge), placés dans un aquarium en verre de 6 litres de capacité rempli au deux tiers avec de l'eau de gîte à mollusques filtrée, d'un $\mathrm{pH}$ voisin de 6,5 et à la température du laboratoire $\left(240-25^{\circ} \mathrm{C}\right)$.

Pour chaque série de tests il esł prévu un aquarium témoin avec 30 mollusques.

Le temps de contact est de 24 heures et il est suivi d'un temps de lavage ou de réanimation de 48 heures. Pendant ce dernier, des feuilles de laitue bouillie sont mises à la disposition des mollusques, et durant toute l'expérience, l'eau des aquariums est oxygénée par un aérateur à bulles.

Ce protocole expérimental est sensiblement celui préconisé récemment par l'Organisation mondiale de la santé, avec cependant cette différence que nous préférons ne pas utiliser l'eau de la canalisation urbaine, à notre avis à déconseiller, car les produits chimiques que l'on y ajoute parfois pour la rendre potable sont quelquefois toxiques pour les mollusques. La seconde ne convient pas non plus à des tests biologiques, car il s'avère que ce milieu n'est pas viable pour les mollusques.

\section{Résultats obtenus in vitro}

Le diméthyldithiocarbamate de zinc agit beaucoup plus lentement sur les mollusques d'eau douce que ne le font les sels solubles de cuivre ou le Bayer 73.

Comme l'ont constaté NOLAN et BOND en 1955, la concentration de 1,5 p.p.m. tue 100 p. 100 des spécimens en expérience.

Des quatre espèces essayées, c'est $L$. caillaudi qui esl la plus sensible à l'action du zirame, puis viennent $B$. pfeifferi gaudi, Bulinus guernei et enfin $B$. senegalensis qui se monitre la plus résistante à ce produit mais qui est tuée à des concentrations voisines de 2 p.p.m.

En ce qui concerne les voies de pénétration ef le mode d'action du zirame chez les mollusques, il est encore un peu trop tôt pour conclure. PAU- 
LINI, CHAIA et FREITAS (1960) parlent d'une pénétration du produit par la voie digestive, mais nous pensons que le zirame agit aussi par contact, car les spécimens placés dans une solution à 10 p.p.m. montrent des signes d'intoxication dès qu'ils sont placés dans ce milieu.

Ces symptômes sont tout d'abord de la fatigabilité avec diminution considérable des réflexes et état hypnotique, contrairement à ce que l'on observe avec les sels solubles de cuivre qui provoquent de l'hyperexcitation avec incoordination motrice.

Dans des solutions très faiblement concentrées $(0,1$ à 0,2 p.p.m.), les mollusques sont immobilisés, le pied rétracté au fond de leur coquille.

Nous avons étudié le pouvair rémanent ef la résistance à la lumière solaire du diméthyldithiocarbamate de zinc au laboratoire, en plaçant B. pfeifferi gaudi et B. guernei dans des aquariums contenant des solutions du produit à 5 ef 10 p.p.m. et laissés en place pendant 45 jours sur le bord d'une fenêtre exposée au soleil pendant tout l'après-midi.

L'activité molluscocide de ce produit se maintient pratiquement in vitro pendant une durée de 30 jours pour s'annuler vers le 45 jour.

Le zirame n'est donc que très lentement détruit par la lumière solaire ef comme, d'autre part, les tests ont été faits dans des aquariums dont le fond était recouvert d'une épaisse couche de matières organiques, il n'est que très lentement absorbé par ces dernières et ne doit pas former avec elles des composés inactifs comme c'est le cas pour les sels solubles de cuivre.

Au sujet de ces derniers, nous signalons qu'il serait tout à fait contre-indiqué de les associer avec le zirame pour essayer d'augmenter son pouvoir molluscocide. Il y a en effet incompatibilité entre ces deux produits et le mélange est totalement inactif contre les mollusques.

L'association zirame-sulfate basique de cuivre, qui est stable, puisque ce sel de cuivre est insoluble dans l'eau, n'apporte aucun avantage et ne nous a donné que des résultats très médiocres, le zirame étant le seul à agir puisqu'il est le seul produit soluble de ce mélange.

Au point de vue du pouvoir ovicide du zirame sur les pontes de mollusques, nous l'avons testé sur B. pfeifferi gaudi et L. caillaudi. L'action du zirame est très lente : mais au bout d'une semaine la croissance des embryons, provenant de pontes traitées avec des solutions à 5 p.p.m. de zirame pendant 24 heures puis mises dans une eau de lavage, est ralentie puis arrêtée, les jeunes mollusques étant dans l'impossibilité de sortir de leurs alvéoles.

\section{Essais faits sur le terrain :}

La nature des gîtes à mollusques que nous avions déjà prospectés au Sénégal, la HauteCasamance mise à part, ne nous a permis qu'une expérimentation dans des mares permanentes ou dans des marigots, (GRÉTILLAT, 1961, a ; 1961, c et 1961, d).

$C^{\prime}$ est ainsi que nous avons pu expérimenter ce produit dans un marigot de la Ferme du Laboratoire national de recherches vétérinaires de Dakar située à Sangalkam, dans la région de la presqu'île du Cap Vert, et dans plusieurs mares et marigots de la région de Tambacounda (Sénégal Oriental).

A la Ferme de Sangalkam dans une eau de $\mathrm{pH}$ 6,2 très riche en matières organiques, avec à la surface de très nombreuses plantes aquatiques du genre Pistia L., et ayant une température de surface de 170 le matin et de $25^{\circ} \mathrm{C}$ le soir, nous avons obtenu les résultats suivants en utilisant du diméthyldithiocarbamate de zinc à 10 p.p.m. :

Le produit a très bien diffusé malgré la présence des nombreuses racines de Pistia.

Par des tests de rémanence contrôlée, (prélèvements d'eau faits toutes les semaines ef ramenés au laborałoire pour contrôle), le milieu est resté toxique pour $L$. caillaudi pendant une période de 35 jours et pour B. pfeifferi gaudi pendant 30 jours.

Les premiers mollusques, des $B$. senegalensis et des $L$. caillaudi, ne sont réapparus que 4 mois et demi après le traitement du marigot.

C'est au cours de cet essai sur le terrain que nous avons pu constater l'action herbicide du zirame sur les Pistia. Ces plantes aquatiques, dont la présence en grand nombre à la surface des plans d'eau est très souvent un gros handicap quand elles encombrent les retenues de barrage, sont d'autre part d'importants gîtes à moustiques, car leurs racines flottantes servent de support respiratoire aux larves de Taeniorhynchus et de Ficalbia.

L'action du zirame sur les Pistio n'est pas immédiate ef ce n'est qu'une semaine après le traitement de l'eau que l'on assiste à un jaunis- 
sement général des callerettes fevillues de ces plantes dont les racines pourries se détachent au niveau du collet. (GRETILLAT, 1961, b).

II est à noter que si le diméthyldithiocarbamate de zinc est phytotoxique pour ces plantes aquatiques, il ne semble avoir aucun effet corrosif sur les feuilles des végétaux terrestres. Une plantation de choux fourragers de la Ferme a été arrosée par aspersion avec l'eau du marigot traité, pendant plus d'un mois sans accident d'aucune sorte.

Dans la région située à l'ouest de Tambacounda (Sénégal oriental), nous avons pu traiter une mare permanente (mare de Panal), à raison de 1 à 1,5 p.p.m. de zirame. Cette collection d'eau de dimensions assez importantes, puisqu'il s'agissait de traiter $25.000 \mathrm{~m}^{3}$ d'eau environ, est un gîte à $B$. guernei. Cet essai nous a permis de constater que dans une equ de $\mathrm{pH}$ voisin de 6,6, très limoneuse, il suffit de 1 à 1,5 p.p.m. de diméthyldithiocarbamate de zinc pour détruire les mollusques d'eau douce.

Dans un autre marigot très fangeux de la même région nous avons utilisé des doses identiques avec des résultats positifs sur B. guernei (marigot de Bountounko).

Ce point d'eau, en voie de desséchement lors de son traitement en février 1961, est un important gîte d̀ Anopheles sp. et cette expérience nous a permis de constater que sur le terrain, le zirame tout comme au laboratoire, est hautement toxique pour les larves de Culicidae (GRÉTILLAT, $1961, a ; 1961, b)$.

C'est en effet au cours de l'expérimentation sur l'activité molluscocide de ce produit in vitro; que nous avons eu la bonne fortune de découvrir l'activité larvicide du diméthyldithiocarbamate de zinc sur les larves de Culex fotigans Wiedemam. Des concentrations de 0,5 p.p.m. suffisent à entraver l'évolution des larves de $1 \mathrm{er}$ stade de ce moustique, mais il faut atteindre 5 et 10 p.p.m. pour tuer celles du stade IV. et les nymphes.

Nous avons enfin essayé le zirame à 5 p.p.m. dans une nappe d'eau, en partie souterraine et très fangeuse, située à une cinquantaine de kilomètres de Tambacounda (mare de Makile).

Cette résurgence, qui est un gîte permanent à B. guernei, a été débarrassée complètement de sa faune malacologique à la suite de ce traitement, et l'activité molluscocide du zirame n'a été en aucune manière diminuée par la présence d'une épaisse couche de vase dans le fond de cette poche d'eau dont le $\mathrm{pH}$ était de 6,5.

\section{Toxicité du zirame pour la faune aquatique.}

Nous l'avons testée au laboratoire sur Tilapia melanopleura et Caraussius auratus.

LLOYD, en 1960, considère que pour les sels de zinc leur toxicité vis-à-vis des poissons peut être évaluée in vitro en prenant comme critère l'arrêt des mouvements respiratoires après 5 heures de contact.

Sur les deux espèces précédentes, les doses toxiques de zirame se situeraient entre 5 à 10 p.p.m. pour des poissons adultes.

Les signes d'intoxication débutent par de l'accélération respiratoire et de l'hyperexcitabilité.

Pour les alevins, les doses toxiques sont beaucoup plus faibles (1 à 2 p.p.m.).

Les batraciens adultes ne semblent pas être sensibles au zirame. Par contre leurs larves (têtards) sont tuées à partir de 5 p.p.m. (essai fait dans une petite poche d'eau de la Ferme de Sangalkam).

Les larves d'Odonates (Libellules) sont très sensibles au zirame ef des doses de 1 à 2 p.p.m. suffisent pour les détruire.

Les coléoptères aquatiques adultes sont insensibles à des doses de 10 p.p.m., ainsi que les nématodes dulcaquicoles.

En résumé, ce sont surtout les formes à respiration branchiale qui seraient tuées par ce dérivé du zinc ; cela confirmerait l'hypothèse de LLOYD (1960), qui fait intervenir l'irritation de la muqueuse branchiale par les sels de zinc comme principale cause dans la toxicité de ces produits pour les poissons.

Toxicité du diméthyldithiocarbamate de zinc pour l'Homme et les animaux domestiques.

Au cours de l'expérimentation sur le lerrain, I'utilisation du zirame sous forme de poudre micronisée n'a demandé aucune précaution spéciale lors de son épandage, si ce n'est celle de ne pas mettre le produit en contact avec les muqueuses ou les yeux.

Les travaux de HODGE, MAYNARD et Coll. (1952), sur souris, lapins et chiens, montrent que 
la dose létale 50 pour ce produit est de l'ordre de $1.250 \mathrm{mg} / \mathrm{kg}$.

Chez le mouton, comme nous avons pu le constater, des doses per os de $25 \mathrm{mg} / \mathrm{kg} / \mathrm{jour}$ pendant une semaine ne déclenche aucun signe d'intoxication.
L'homme et les animaux domestiques peuvent donc utiliser sans danger comme boisson des eaux traitées même avec 10 p.p.m. de zirame. La manipulation et l'épandage de ce produit ne présentent pratiquement aucun risque d'intoxication de la part des ufilisateurs.

TABLEAU COPAPATI des Dropritétés de différents nolluscocides.

\begin{tabular}{|c|c|c|c|c|c|c|c|c|c|c|}
\hline Iiolluscocides & 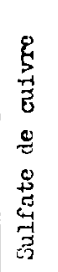 & 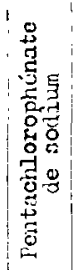 & 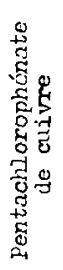 & 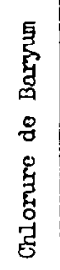 & $\begin{array}{l}A \\
= \\
0 \\
= \\
\therefore\end{array}$ & 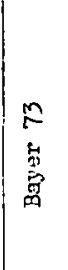 & 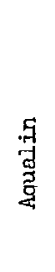 & $\begin{array}{l}\mathrm{M} \\
\mathrm{N} \\
\mathbb{N} \\
\mathrm{H} \\
\mathrm{H}\end{array}$ & 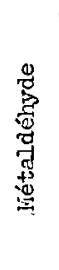 & 兽 \\
\hline Forme d'euploi & Cr. & $\mathrm{P}-\mathrm{T}$ & $P$ & Cr. & $\mathrm{P}$ & $P$ & $\mathrm{~L}$ & $\mathrm{P}$ & $\mathrm{P}$ & $\mathrm{P}$ \\
\hline Solubilité dans $1^{\prime}$ eau & oui & oui & non & oui & oui & oui & oui & oui & non & oui \\
\hline $\begin{array}{c}\text { Dose lótale mollusque au laboretoire }(24 \mathrm{~h}) \\
\text { en parties par million }\end{array}$ & $\begin{array}{l}1 \\
\text { à } \\
5\end{array}$ & 5 & $\begin{array}{c}1 \\
\text { à } 8\end{array}$ & $\begin{array}{l}25 \\
30\end{array}$ & $\begin{array}{l}0,5 \\
\text { a } 3\end{array}$ & $\begin{array}{l}0,2 \\
\text { à } 0,5\end{array}$ & 3 & $\begin{array}{c}0,2 \\
\mathrm{a} 0,5\end{array}$ & - & 1.5 \\
\hline $\begin{array}{c}\text { Dosc létale mollusque sur le terrain } \\
\text { en parties par million }\end{array}$ & 20 & 5 & 15 & - & & 0,4 & 10 & - & - & 1 \\
\hline Ovicide & non & i & oui & - & our & oui & תi & oui & non & oui \\
\hline Cercaricide & non & oui & - & - & oui & oui & i & non & non & - \\
\hline Risictance aux R.U.V. & oui & non & - & non & - & nor. & - & non & - & oui \\
\hline Faucardage conseillé & oui & oui & oui & - & oui & ت نגיס & - & non & - & non \\
\hline Activité en milieu boueux & & & & - & - & $\longrightarrow$ & & $\rightarrow$ & - & $\rightarrow$ \\
\hline Influence du pH & $\alpha i$ & nont & non & non & - & non & - & - & - & non \\
\hline Larvicide (noustiques) & non & ron & Eon & non & non & non & - & - & non & oui \\
\hline Ferbicide (plantes aquatiques) & non & nor: & non. & non: & non & non & oui & non & - & oui \\
\hline Phytotoxicité pour plantes cultivées & non & $\alpha i$ & - & $\boldsymbol{-}$ & non & non & non & non & - & non \\
\hline Ichtyotoricité & non & خندم & $\sigma i$ & nor. & oui & oui & itur & oui & non & oui \\
\hline Moxicité batraciens & r.on & $\sigma i$ & oii & - & oui & oui & - & - & - & non \\
\hline Foxicité por I'hoxwe ax doses utiiisées & non & non & & nor. & non & non & non & non & non & non \\
\hline Facilité d'emploi & ousi & iنس & oui & oui & ousi & oui & non & oui & non & oui \\
\hline Prix de reviert de l'opération & & $\because$ & $\therefore$ & - & - & - & $\uparrow$ & - & $\uparrow$ & \\
\hline
\end{tabular}

Légende des ajréviations :

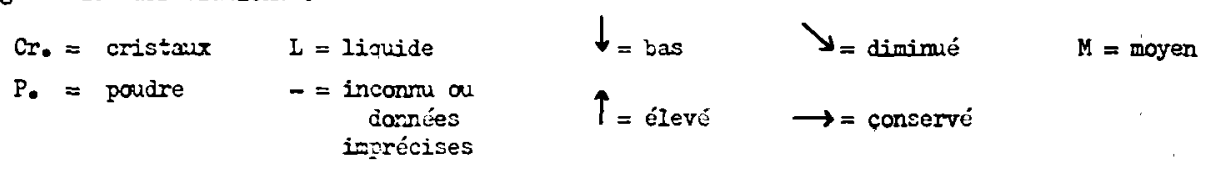




\section{DISCUSSION ET CONCLUSION}

Comme on peut s'en rendre compte, il n'existe pas à l'heure actuelle de produit molluscocide omnivalent pouvant agir dans n'importe quel gîte à mollusques avec le maximum d'efficacité.

Pour chaque région à assainir, toute action d'envergure doit être précédée de plusieurs enquêtes sur le terrain qui doivent préciser la faune et la flore des gîtes à traiter et leurs conditions physico-chimiques: $(\mathrm{pH}$ de l'eau; nature du fond, courant, débit, etc...).

Les nombreux échecs, enregistrés parfois dans certains pays où on a utilisé contre les mollusques d'eau douce un produit qui pourtant avait fait ses preuves en d'autres lieux, viennent très souvent de ce que l'on n'a pas tenu compte de ces facteurs qui sont primordiaux.

Au sujet des programmes de lutte antimollusques à mettre sur pied dans certaines régions de l'Ouest africain, particulièrement infestées par des affections à trématodes de l'Homme et des animaux domestiques, (schistosomiase humaine vésicale ou intestinale, schistosomiase bovine, distomatose des ruminants), nous pensons qu'au point de vue économique, il serait intéressant de coordonner et d'associer les efforts du Service de Santé et ceux du Service de l'Elevage. Très sou- vent en effet, les hôtes intermédiaires de ces différentes affections coexistent dans les mêmes gîtes où il suffirait d'une intervention unique pour les détruire et couper le cycle évolutif de ces divers parasites.

Comme il serait trop long de discuter des avantages et des inconvénients présentés par les différents produits anti-mollusques en les comparant les uns avec les autres nous les donnons sous forme d'un tableau général.

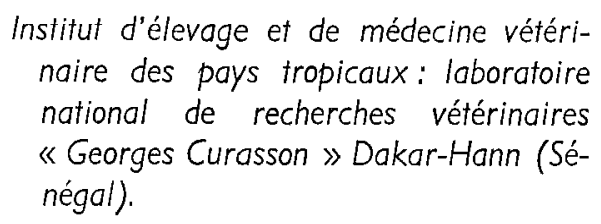

\section{REMERCIEMENTS}

Nous remercions Monsieur le professeur G. RANSON du Muséum d'Histoire Naturelle de Paris d'avoir bien voulu nous déterminer les souches de nos élevages de mollusques ayant servi d̀ nos expériences.

Nous remercions aussi la Société Rhône-Poulenc de Paris qui nous a fourni le diméthyldithiocarbamate de zinc nécessaire à nos essais au laboratoire et sur le terrain.

\section{SUMMARY}

\section{Distomatosis and Bilharziosis of domestic animals - Prophylaxis by Snail Control.}

The treatment of distomatosis and schistomiasis of ruminants under ranching conditions is practically impossible. Prophylaxis by action against the snail vector gives better results. Drainage and other methods of drying swamp areas limits or eliminates their proliferation, and their destruction can be accomplished by the introduction of swans, ducks, crustacae or the use of chemicals.

This latter method is the most effective according to the author, who discussed the advantages and disadvantages of the principal molluscides, and in particular dimethyldithiocarbamate of Zinc, (Zirame) which he considers of considerable value as a result of his experiments carried out at Dakar.

Before spreading it, however, it is necessary to make a survey of the area to determine the flora, fauna and the physical and chemical conditions, e. g. pH of the water, nature of the bottom, current, flow, etc., of the mollusc sites. Coordination is necessary between veterinary and public health services. 


\section{RESUMEN}

Distomatosis y bilarciosis de rumiantes domésticos. Su profilaxia por la lucha contra los moluscos.

El tratamiento de la distomatosis y schistosomiasis de los rumiantes en una ganadería extensiva est prácticamente imposible. Las medidas profilacticas que actúan sobre el molusco vector permiten obtener mejores resultados : mediante trabajos de drenaje y de desecación, se puede limitar o impedir su proliferación ; para destruirle se pueden introducir especies animales (gansos, patos, crustáceos) o utilizar productos químicos. Este último procedimiento es el más eficaz, para el autor, que expone las ventajas e inconvenientes de los principales productos empleados. El autor describe particularmente las investigaciones que él ha realizado en Dakar sobre las propiedades de uno de estos productos, el dimetiditiocarbamato de zinc que le parece ser particularmente interesante.

Pero antes de una acción de envergadura, es preciso realizar investigaciones sobre el terreno para precisar la flora, fauna y las condiciones fisico-químicas $(\mathrm{pH}$ del agua, naturaleza del fondo, corrientes, caudal, etc.) de los albergues de moluscos. Es necesaria también una acción conjunta de los servicios ganaderos y sanitarios.

\section{BIBLIOGRAPHIE *}

AGUIRRE (G. H.), SILVA (J.F.) et TAVARES (M. D.) 1955. - Sulfato de cobre como planorbicida ; seu emprêgo no Setor Bahia do Servico Nacional de Malaria. Rev. Brasil. Molar. e Doencos tropiciais, 7 (2) : 235-43.

ALVES (W.) 1956. - Un problème particulier concernant l'écologie des mollusques. Groupe d'études sur l'écologie des mollusques hôtes intermédiaires de la bilharziose. O. M. S. Paris 3-9 octobre 1956, no 12.

ANONYME 1958. - Process for controlling aquatic vegetation with F-98 aquatic herbicide Shell Development Company, Modesto, California (processed report) 9 pages.

BARBAGALLO (R. P.) 1896. - Contributo allo studio della Bilharzia crassa (Sons.) in Silicia, Atti Accad. Gioenia Sci. not. Catania, n. 5.. (59) : 15-16.

\footnotetext{
* Les références dont le titre est suivi de la mention : «Document de travail non publié », correspondent à des rapports de travaux que l'Organisation mondiale de la Santé à Genève a bien voulu mettre à notre disposition. A cet égard nous remercions tout particulièrement Monsieur le médecin-chef N. ANSARI (Maladies endémoépidémiques, division des maladies transmissibles, O.M. S. Genève) d'avoir eu l'amabilité de nous faire parvenir tous ces documents.
}

BARNES (J. M.) 1960. - Pentachlorophénol et pentachlorophénate de soude. (Original en anglais) Comité d'experts de la bilharziose Genève 26-IX-60, W. H. O.BILHARZ/23, 23-V-60 (Document de travail non publié).

BAYER (F. A. H.) 1954. - Larval trematodes found in some fresh water snails: a suggested biological method of bilharzia control. Trans, roy. Soc. trop. Med. Hyg., 48 : 418-8 BERRY (E. G.) 1960. - L'utilisation des molluscocides en Afrique. (Orig. en anglais). Symposium africain sur la Bilharziose. Lourenço Marques 30-III-60, W. H. O. AFR/BILHARZ/6, 16-ll-60 (Document de travail non publié.) BIJAN (H.) et DESCHIENS (R.) 1956. - Action des sels de baryum sur les mollusques vecteurs de bilharzioses. Bull. Soc. Path. exot., $49 ; 455-8$.

BIOCCA (E.) ef LEROUX (P. L.) 1952. - The molluscan host of Schistosoma bovis and other Trematodes in Sardinia. Trans. roy. Soc. trop. Med. Hyg., $46: 12$.

BRUMPT (E.) 1929. - Cycle évolutif de Schitosoma bovis (Bilharzia crassa). Infection spontanée de Bulinus contortus en Corse. C. $R$. Acod. Sci., 189 : 879.

BRUMPT (E.) 1930. - Cycle évolutif complet du Schistomosa bovis. Infection naturelle en Corse et infection expérimentale de Bulinus contortus. Ann. Parosit. hum. comp., 8 : 17-50. 
BLAIR (D. M.) 1960. - Dangers de l'utilisation et de la manipulation du pentachlorophénate de sodium comme molluscocide (Orig. en anglais) Comité d'Experts de la Bilharziose, Genève 26-IX-60, W.H.O./BILHARZ/25,

1-VI-60 (Document de travail non publié).

CHERNIN (E)., MICHELSON (E.H.) et AUGUSTINE (D. L.) 1956. - Studies on the biological control of schistosome bearing-snails. II-Control of Australorbis glabratus populations by the leech Helobdella fusca under laboratory conditions. Amer. J. Trop. Med. Hyg., 5 (2) : 308-314,

CORROLLER le (Y.) 1960. - Surl'action molluscocide du chlorure de baryum sur Biomphalaria (Planorbis) boissyi. Bull. Soc. Path. exot, 53 : 798-802.

COUMBARAS (A.) 1960. - La bilharziose dans le Sud-tunisien. Etude particulière du Nefzooua. (Région de Kebili-Douz). 17 phot., 1 carte. Arch. Inst. Past. Tunis, 37 (3) : 31335.

DESCHIENS (R.) 1954. - Mécanisme d'action léthale de Cypridopsis hartwigi sur les mollusques vecteurs de bilharzloses. Bull. Soc. Path. exot., 47 : 399-401.

DESCHIENS (R.), AYAD(N.) etCOROLLERLE(Y.) 1960. - Molluscicides à action élective ef molluscicides de contact dans la prophylaxie des bilahrzioses. Comité d'experts de la bilharziose, Genève, WHO/BILHARZ/50, 12-IX-60 (Document de travail non publié).

DESCHIENS (R.), DESCHANGE (M.) et VERMEIL C. (1955) - Action prédatrice des crabes d'eau douce du genre Pofamon sur les mollusques vecteurs de bilharzioses. Bull. Soc. Path. cxot., 48 : 203-7.

DESCHIENS (R.) et LAMY (L.) 1954. - Action prédatrice des écrevisses des genres Astacus et Cambarus sur les mollusques vecteurs de bilharzioses. Bull. Soc. Path. exot., 47 : 809-12.

DESCHIENS (R.) et LAMY (L.) 1955. - Préhension ef ingestion des mollusques vecteurs de bilharzioses par les écrevisses du genre Cambarus. Bull. Soc. Path. exot., 48 : 201-3.

DESCHIENS. (R.) et MOLINARI (V.) 1956. - Sur l'action molluscocide de l'oxyde de zinc et de l'aldéhyde méthylique. Bull. Soc. Path. exot., $49: 1111-3$.
DESCHIENS (R.) et MOLINARI (V.) 1957. - Sur l'action molluscocide de la grenaille de zinc. Bull. Soc. Poth. exot., 50 (1) : 62-65.

DESCHIENS (R.), MOLINARI ef BERTRAND (D). 1957. - Sur l'action molluscocide de « l'eau de zinc». Bull. Soc. Path. exot., 50(1) : 59-61.

DIAS (E.) 1953. - Nova possibilidade de combate aos moluscos transmissores dos esquitossomoses. Emprêso Editora «O ECO», Bambui, 22 pages.

DIAS (E.) 1954. - Guerra bacteriológica contra os hospedeiros intermediários da esquistossomose humana. Mem. Inst. Oswaldo Cruz, 52 : 315-327.

DIAS (E.) et DAWOOD (M. M.) 1955. - Preliminary trials on the biological snail control with Bacillus pinotti in Egypt. Mem. Inst. Oswaldo Cruz, $53: 13-29$.

DOBROVOLNY (C. G.) et BARBOSA (F.S.) 1953. - Fields trials of sodium pentachlorophenate as a molluscicide in flowing waters in Brazil. Publ. avuls. Inst. Aggon. Magalhâes 2 (10) : 121-57.

ENIGK VON (K.) 1956. - Die Bekâmpfung des Leberegelbefalles. Deutsche Teirârzl. Wochens., 63 (41-42) : 425-8.

ENIGK VON (K.) et DÜWEL (D.) 1960. - Die Durchführung des Bekâmpfung der Leberegelschnecke Galba truncafula (Limnaeidae). Mschr. Tierheilkunde, 12 (9) : 259-80.

FFRGUSON (F. F.) et PALMER (I. R.) 1960 . Mollusciciding for bilharziasis control in Puerto-Rico. Comité d'experts de la bilharziose, Genève. WHO. BILHARZ. 53, 15-1X-60 (Document de travail non publié).

FERGUSON (F. F.), RICIHARDS (C. S.) et PALMER (J. R.) 1960. - Control of Australorbis glabratus by Acrolein in Puerto-Rico. Comité d'experts de la bilharziose, Genève. WHO. BILHARZ/54, 15-IX-60 (Document de travail non publié).

FOSTER (R.) TEESDALE (C.) etPOULTON (G. F.) 1960. - Trials with à new molluscicide. Bull. Org. mond. Santé, 22 : 543-8.

FREITAS DE (J. R.) 1959. - Acao do moluscicida Bayer 73 sobre os ovos de Taphius (Australorbis) glabratus. IX Reuniâo Anual S.B.P.C. Salvador (Bahia). 
GILLET (J.) et BRUAUX (P.) 1960. - Essai en laboratoire avec un nouveau molluscocide, «Bayer 73 ». Symposium africain sur les bilharzioses, Lourenço-Marques, WHO/AFR/BILHARZ/10, 22-111-60 (Document de travail non publié).

GILLET (J..) BRUAUX (P.) ef MANNAN (P.) 1960...-Premier bilan (1956-1959) de la lutte contre la bilharziose menée par la mission médicale établie dans la plaine de la Ruzizi, Congo Belge (Mission médicale de la Ruzizi). Symposium africain sur les bilharzioses, Lourenço Marqués WHO/AFR/BILHARZ/12, 31III-60 (Document de travail non publié).

GINDY el (N. S.) 1953. - Factors affecting the stability of copper sulphate solution in Nile water. J. roy. egypt. Med. Assoc., 36 : 309-12.

GINDY el (M. S.) 1957. - Field Studies on the action of copper sulphate as a molluscicide for control of the snail vector of Schistosomiasis in Egypt. J. egypt. Med. Assoc., 40 (2) : 111-21.

GÖNNERT (R.) 1960. - Results of laboratory and fields trials with molluscicide Bayer 73. Comité d'experts de la bilharziose, Genève. WHO/BILHARZ/56, 26-IX-60 (Document de travail non publié).

GÖNNERT (R.) et SCHRAUFSTAETTER (E.) 1958. - A new molluscicide compound, Molluscicide Bayer 73. 6e Congrès intern. Méd. trop. Malaria, Lisbonne, sept. 1958.

GRASSI (C. B.) et ROVELLI (G.) 1888. - La Bilharzia in Sicilia. Gazz. Med. Ital. Lomb., XLVIII, 9: Sér., I (29) : 281.

GRÉTILLAT (S.) 1961 (a). - Un nouveau molluscocide, le diméthyldithiocarbamate de zinc (Zirame). Bull. Org. mond. Santé (sous presse).

GRÉTILLAT (S.) 1961 (b). — Un molluscocide actif contre les formes larvaires de Culicidae, le diméthyldithiocarbamate de zinc. Bull. Org. mond. Santé (sous presse).

GRÉTILLAT (S.) 1961 (c). - Epidémiologie de la bilharziose vésicale au Sénégal Oriental. Observations sur l'écologie de deux bulins: Bulinus guernei ef Bulinus senegalensis. Bull. Org. mond. Santé (sous presse).
GRÉTILLAT (S.) 1961 (d). - Note préliminaire sur l'épidémiologie de la distomatose bovine au Sénégal. Rev. Elev. Méd. vét: Pays trop. 14 (3) : 283-91.

HALAWANI (A.) 1954. - Control of schistosomiasis by molluscacides. Report on the Molluscacides Sodium Pentachlorophenate and Dinitro-O-Cyclohexylphenol (D.C.H.P.) J. egypt. Med. Assoc., 37 (6) : 581-612.

HERDT (J. R.), LOOMIS (L. N.) et NOLAN (M. O.) 1951. - Effect on calves of prolonged oral administration of three potential molluscacides. Publ. Health Rep. Wash., 66 (41) : 1313-7.

HODGE (H. C., MAYNARD (E. A.) et COLL. 1952. - Acute and short-term oral toxicity tests of ferric dimethyldithiocarbamate (ferbam) and zinc dimethyldithiocarbamate (ziram). J. amer. Pharm. Assoc., 4l (12) : 662.

JOVE (J. A.) 1956. - Use of molluscicides in the control of Bilharziasis in Venezuela. Equipment and methods of application. Bull. Org. mond. Sté, 14 (4) : 617-38

KOMIYA (Y.), HOSAKA (Y.) ef YASURAOKA (K.) 1960. - Normalisation d'une technique d'épreuve de la sensibilité des mollusques Onchomelania au pentachlorophénate de sodium. (Orig. en anglais) Comité d'experts de la bilharziose, Genève. WHO/BILHARZ/35, 21-VI-60 (Document de travail non publié).

KUNTZ (R. E.) et STIREWALT (M. A.) 1950. Laboratory evaluation of two dinitro-phenols as molluscacides. Proc. Helminth. Soc. Wash., 17(2) : 95-102.

KUNTZ (R.E.) et WELLS (W.H.) 1951. - Laboratory and Field evaluations of two DinitroPhenols as molluscacides of control of Schistosome Vectors in Egypt with emphasis on importance of temperature. Amer J. trop, Med. Hyg., 31 (6) : 784-824.

LAGRANGE (E.) 1951. - L'action oligodynamique sur les Planorbes. C. R. Soc. Biol., Paris, 145 : 458-459.

LAGRANGE (E.) 1953. - La lutte biologique contre les Planorbes. Ann. Soc. belge Méd. trop., 33 : 227-236.

LE ROUX (P.L.) 1929, (a). - Notes of the life cycle of Schistosoma mattheei and observations 
on the control and eradication of Schistosomiasis in Man and Animals. 15 th. Ann. Rept. Director. Vet. Serv. Un. S. Afr., 407-438.

LE ROUX (P.L.) 1929, (b). - Remarks on the habits and pathogenesis of Schistosoma mattheei, together with notes on the pathological lesions observed in Scheep. 15 th. Ann. Rept. Director Vet. Serv. Un. S. Afr., 347-406.

LEUCKART (R.) 1886. — Zur Entwickelungsgeschichte des Leberegels. Zool. Anz. Leipzig, 4; 641-646.

LLOYD (R.) 1960. — The toxicity of zinc sulphate to rainbow trout. Ann. Appl. Bio, 48 (1) ; 84-5.

MALEK (A. E.) 1960 (a). - Bilharziasis control in agricultural pumschemes in the Sudan. (Textes en anglais et en français) Symposium africain sur les bilharzioses. Lourenço-Marquès, mars 1960 WHO/BILHARZ/4., 22-11I-60. (Document de travail non publié).

MALEK (E. A.) 1960 (b). - Les barrières chimiques dans la lutte contre les bilharzioses (original en anglais) Comité d'experts de la bilharziose, Genève. WHO/BILHARZ/36, 25-VII-60, (Document de travail non publié).

MARILL (F. G.) 1960 (a). - Rapport sur une enquête relative à l'épidémiologie des bilharzioses en Mauritanie. Mission d'expertsSecrétariat d'état aux relations avec les états de la Communauté. Direction de la coopération technique (avril-octobre) (non publié) 26 pages.

MARILL (F. G.) 1960 (b). - Rapport annexe sur l'épidémiologie des bilharzioses en Mauritanie. Ibid (avril-octobre) (non publié) 7 pages.

NAGUIB AYAD (1960). - The use of molluscicides in the Egyptian Region of U. A. R. Comité d'experts de la bilharziose, Genève. WHO/BILHARZ/51, 12- IX-60 (Document de travail non publié).

NOLAN (M. O.) et BOND (H. W.) 1955. Results of laboratory screening tests of chemical compounds for molluscacidal activity. III. Derivatives of abietic acid. Amer. J. trop. Med. Hyg.., 4 : 152-155.

OLIVER-GONZALEZ (J.), BAUMAN (P. M.) et BENENSON (A.S.) 1956. - Effect of the snail Marisa cornuarietis on Australorbis glabratus in natural bodies of water in Puerto-Rico. Amer. J. trop. Med. Hyg., 5 : 290-6.

OLIVER (L.) ef HASKINS (W. T.) 1960. - The effects of low concentrations of sodium pentachlorophenate on the fecundity and egg of Australorbis glabratus. Amer. J. trop. Med. Hyg., 9 : 199-205,

PAULINI (E.) 1956. - Da applicaçao de moluscocidas. II. - Preparo de Tijolos de pentachlorofenato de sódio. Rev. Brasil. Malar., 8: 491-2.

PAULINI (E.), CHAIA (G.) et FREITAS (J. R.) 1960. - Essais de nouveaux molluscicides. (Rhodiacid et Bayer 73) Comité d'experts de la bilharziose, Genève. WHO/BILHARZ 42. 23-VIII-6O (document de travail non publié).

PESIGAN (T. P.) et MASILUNGAN (V. A.) 1960. Studies on Schistosomiasis : Experiments on the chemical control of Oncomelania quadrasi Snails. J. Philippine Med. Ass., 26 (1) : 17-30.

POISSON (H). 1929. - Note sur la distomatose des moutons à Madagascar. Bull. Soc. Path. exot., 22 : 521.

PORTER (A.) 1938. - The larval trematoda found in certain South African Mollusca, vith special reference to schistosomiasis (Bilharziasis). Publications of the South African Institute for Medical Research, no XLII (vol. VIII), 492 pages, 83 figures, 1 carte ef une figure hors texte.

RITCHIE (L.S.) et MC LLEN (D.B.) 1960. - Observations sur les essais de molluscicides effectués en Extrême-Orient sous les auspices de l'Armée des Etats-Unis. (Original en anglais) Comité d'experts de la bilharziose, Genève. WHO/BILIIARZ, 25-VII-60 (Document de travail non publié).

SHIFF (C. J.) 1960 (a), - Spraying from the air of a lake margin with sodium pentachlorophenate. Comité d'experts de la bilharziose, Genève. WHO/BILHARZ/5b, 15-1X-60 (Document de travail non publié).

SHIFF (C. J.) 1960 (b). - Essais d'un nouveau molluscicide, le Bayer. 73, en Rhodésie du Sud. (Original en anglais) Comité d'experts de la bilharziose, Genève. WHO/BILHARZ/29, 21-VI-60 (Document de travail non publié). 
THOMAS (A. P. W.) 1881. - Report of experiments on the development of liver fluke (Fas ciola hepatica). Quart. J. Micr. Sci. N. S. 23 ; 99-133.

VAN DEN BERGHE (L.) 1934. - Les schistosomiases humaines et animales au Katanga. Ann. Soc. belge Méd. trop., 16 (3) : 313-74.

VAN OVERBEEK (J.), HUGHES (W. J.) et BLONDEAU (R.) 1959. - Acrolein for the controof water weeds and disease carrying water snails. Science, 129 : 335-6.

WEBBE (G:) 1960. - Essais en laboratoire ef sur le terrain d'un nouveau molluscicide, le
Bayer 73 au Tanganyika. (Original en anglais) Symposium africain sur les bilharzioses, Lourenço-Marqués. WHO/BILHARZ 11. 30-III-60 (Document de travail non publié). WILLIAMS (J. E.), MOON (A. P.), OTORI (Y.), FRICK (L.P.) et RITCHIE(L. S.) 1955. - Applications of molluscacides against juvenile Oncomelania nosophora for repopulation control. J. Parasit., 4I (6), section 2 : 27 (suppl.).

WOLFS J. ef DEVIGNAT (R.) 1949. - Note sur la lutte contre la bilharziose à Costermansville. Ann. Soc. belge. Méd. trop., 29 (4) ; 557 565. 